\title{
Factors predicting pneumonia in patients admitted with spontaneous supratentorial intracerebral hemorrhage in western region of Nepal.
}

\author{
Subhash Yogi ${ }^{1}$, Joshan Neupane ${ }^{2}$, Aabishkar Bhattarai ${ }^{3}$, Bijaya Karki ${ }^{4}$, Benju Tilija Pun ${ }^{3}$ \\ ${ }^{1}$ Consultant Pulmonologist, ${ }^{2}$ Consultant Neurosurgeon, Nepalgunj Medical College and Teaching Hospital, ${ }^{3}$ Medical Officer, \\ Metrocity Hospital and Research Center, Pokhara, Nepal, ${ }^{4}$ Medical Officer, Birat Medical College Teaching Hospital, Nepal.
}

Received: December 16, 2020

Accepted: February 01, 2021

Published: March 30, 2021

Cite this paper:

Yogi S, Neupane J, Bhattarai A, Karki B, Pun BT. Factors predicting pneumonia in patients admitted with spontaneous supratentorial intracerebral hemorrhage in western region of Nepal. Journal of Brain and Spine Foundation Nepal. 2021:2(1):3136.

\author{
Correspondence: \\ Subhash Yogi \\ Consultant Pulmonologist \\ Nepalgunj Medical College and Teaching Hospital, Nepalgunj, Nepal. \\ Email: subhashyogi@hotmail.com \\ ORCID: https://orcid.org/0000-0002-0683-3201
}

\begin{abstract}
:
Introduction: Introduction: Despite the well-documented association of stroke-associated complications and infections with increased mortality, morbidity and worse long-term outcome, there are only limited data available on independent predictors of pneumonia in patients with acute intracerebral hemorrhage. In this study, our objective was to evaluate risk factors and comorbid conditions associated with the diagnosis of hospital acquired pneumonia in the patients admitted with spontaneous intracerebral hemorrhage and to determine the independent predictors of pneumonia in these patients. Methods: A retrospective analysis was done in patients admitted in Intensive Care Unit of Nepalgunj Medical College with spontaneous intracerebral hemorrhage. Various clinic-demographic parameters were tested for association with pneumonia or no pneumonia group using chi square or student's "t" test. Results: A total of 117 patients, 86 men (73.5\%) and 31 women (26.5\%) with spontaneous intracerebral hemorrhage were included in the study. There were $36(30.77 \%)$ patients with pneumonia. The association Diabetes mellitus $(p<0.01)$, COPD ( $p<0.01)$, smoking $(p<0.01)$, mean GCS $(p<0.01)$, ICH volume $(p=0.01)$, ICH score $(p<0.01)$, operated status $(p<0.01)$, Ganglionic $(<0.01)$, Brain stem $(\mathrm{p}=0.03)$ and Ventricular $(\mathrm{p}=0.01)$ location of hematoma was statistically significant with pneumonia. Outcome in terms of MRS at discharge was also poorer for patients with pneumonia $(\mathrm{p}=0.01)$. Conclusion: Diabetes mellitus, COPD, mean GCS, ICH volume, ICH score, operated status, higher MRS score were associated with increased risk of pneumonia in ICH. Similarly, ganglionic hematoma, brainstem hematoma and those having intraventricular hemorrhages are also associated with increased risk of pneumonia. Outcome in patients in terms of MRS were also poorer in those who had pneumonia.

Keywords: Hemorrhagic stroke, Pneumonia, Intensive care unit, Risk factors.
\end{abstract}

\section{Introduction:}

Pneumonia is among the most common medical complications after stroke, with an estimated incidence ranging from $2.4 \%$ to $47 \% .^{1}$ Despite the welldocumented association of stroke-associated complications and infections with increased mortality, morbidity and worse long-term outcome, there are only limited data available on independent predictors of pneumonia in patients with acute intracerebral hemorrhage. The identification of early predictors is of paramount importance for the treating neurologist, neurosurgeons and physicians, so that specific management plan and therapeutic modalities can be applied to patients with high risk of morbidity and 
mortality. In this observational study, our objective was to evaluate risk factors and comorbid conditions associated with the diagnosis of hospital acquired pneumonia in the patients admitted with spontaneous intracerebral hemorrhage and to determine the independent predictors of pneumonia in those patients.

\section{Methods:}

A retrospective analysis was done in patients admitted in Intensive Care Unit (ICU) of Nepalgunj medical College Teaching Hospital, Nepal with spontaneous supratentorial hypertensive intracerebral hemorrhage (ICH) in between June 2018- June 2019. Records of patients were retrieved in terms of demographics (age, gender), clinical parameters (seizure, dysarthria, hypertension, COPD, Diabetes, known cardiac diseases, smoking, alcohol, operated cases, volume of bleed, location of bleed, ICH score, GCS at presentation, pneumonia etc.) and outcome in terms of death and Modified Rankin Scale (MRS). Pneumonia in this study was defined according to the criteria of US Center for Disease Control and Prevention for hospital-acquired pneumonia. ${ }^{2}$ Presence of consolidations or infiltrates in chest x-ray along with fever, cough, leukocytosis, tachypnea, hypoxia, crackles on examination, purulent sputum and positive sputum culture in the patient's record were the parameters considered for diagnosis of pneumonia. Patients with preexisting pneumonia at admission and mentioned in records or suspicious according the aforementioned findings were excluded from the study. Similarly, patients with intracerebral hemorrhage likely due to other caused e.g. trauma, subarachnoid hemorrhage due to aneurysms, intracerebral hemorrhages due to sinus venous thrombosis, tumors, arterio-venous malformations, coagulopathy etc. were also excluded from the study. Patients less than 18 years, those with incomplete or lost records, who left against medical advices without completion of treatment and who died within first 3 days of admission were excluded from the study. Patients with ICH were divided in two groups according to presence of pneumonia; Pneumonia and No Pneumonia groups. Various parameters as described above were tested for association with pneumonia and no pneumonia groups.

Statistical analysis was done using IBM SPSS 21.0 software. All the continuous variables were presented as mean \pm SD (standard deviation) and analyzed using student's $t$ test whereas categorical data were presented as percentage and analyzed using chi square/Fischer Exact test (when at least one of the expected values in a cell $<5$ ). $\mathrm{P}$ value $<0.05$ was considered as significant.

\section{Results:}

A total of 117 patients, 86 men (73.5\%) and 31 women (26.5\%) with ICH were included in the study. The mean age was $62.91 \pm 13.84$ years. Thirteen out of 117 (11.10\%) patients had documented seizure and $34.2 \%$ (40/117) of patient developed dysarthria. Fifty-nine out of 117 (50.4\%) were diagnosed with hypertension and took some form of antihypertensive medications. Sixteen patients (13.7\%) had been diagnosed with Diabetes Mellitus; $62.5 \%$ (10/16) of those developed pneumonia during the hospital stay, and the association was statistically significant $(\mathrm{p}<0.01)$. Out of total patients under study, $10.3 \%$ (12/117) were diagnosed and treated for some forms of cardiac disease, 13 patients (11.1\%) had previous stroke and $58.1 \%$ (68/117) of the patient consumed alcohol in some forms. The history of COPD was found in $20.5 \%$ (24/117) of study population and $58.33 \%$ (14/24) of total COPD patient developed pneumonia during the hospital stay; $24.85 \%$ (29/117) of study population smoked, among those 58.62\% (17/29) developed pneumonia in their hospital stay; both of which were statistically significant $(p<0.01)$. 
Table1. Various clinic-demographic parameters versus occurrence of pneumonia

\begin{tabular}{|c|c|c|c|c|c|}
\hline S.N. & Parameters & Pneumonia & No Pneumonia & Total & $\mathrm{P}$ value \\
\hline 1 & Age & $59.28 \pm 14.25$ & $64.52 \pm 13.43$ & $62.91 \pm 13.84$ & 0.06 \\
\hline \multirow[t]{2}{*}{2} & Male & $30(25.6 \%)$ & $56(47.9 \%)$ & $86(73.5 \%)$ & 0.11 \\
\hline & Female & $6(5.1 \%)$ & $25(21.4 \%)$ & $31(26.5 \%)$ & \\
\hline \multirow[t]{2}{*}{3.} & Seizure & $6(5.1 \%)$ & $7(6 \%)$ & $13(11.1 \%)$ & 0.22 \\
\hline & No Seizure & $30(25.6 \%)$ & $74(63.2 \%)$ & $104(88.9 \%)$ & \\
\hline \multirow[t]{2}{*}{4} & Dysarthria & $13(11.1 \%)$ & $27(23.1 \%)$ & $40(34.2 \%)$ & 0.77 \\
\hline & No Dysarthria & $23(19.7 \%)$ & $54(46.2 \%)$ & $77(65.8 \%)$ & \\
\hline \multirow[t]{2}{*}{5} & Hypertension & $20(17.1 \%)$ & $39(33.3 \%)$ & $59(50.4 \%)$ & 0.46 \\
\hline & No Hypertension & $16(13.7 \%)$ & $42(35.9 \%)$ & $58(49.6 \%)$ & \\
\hline \multirow[t]{2}{*}{6} & Diabetes & $10(8.5 \%)$ & $6(5.1 \%)$ & $16(13.7 \%)$ & $<0.01$ \\
\hline & No Diabetes & $26(22.2 \%)$ & $75(64.1 \%)$ & $101(86.3 \%)$ & \\
\hline \multirow[t]{2}{*}{7} & Cardiac Diseases & $4(3.4 \%)$ & $8(6.8 \%)$ & $12(10.3 \%)$ & 1.00 \\
\hline & No Cardiac Diseases & $32(27.4 \%)$ & $73(62.4 \%)$ & $105(89.7 \%)$ & \\
\hline \multirow[t]{3}{*}{8} & Previous Stroke & & & & \\
\hline & Yes & $3(2.6 \%)$ & $10(8.5 \%)$ & $13(11.1 \%)$ & 0.75 \\
\hline & No & $33(28.2 \%)$ & $71(60.7 \%)$ & $104(88.9 \%)$ & \\
\hline \multirow[t]{2}{*}{9} & COPD & $14(12 \%)$ & $10(8.5 \%)$ & $24(20.5 \%)$ & $<0.01$ \\
\hline & No COPD & $22(18.8 \%)$ & $71(60.7 \%)$ & $93(79.5 \%)$ & \\
\hline \multirow[t]{2}{*}{10} & Smoking & $17(14.5 \%)$ & $12(10.3 \%)$ & $29(24.8 \%)$ & $<0.01$ \\
\hline & No Smoking & $19(16.2 \%)$ & $69(59.0 \%)$ & $88(75.2 \%)$ & \\
\hline \multirow[t]{2}{*}{11} & Alcohol & $20(17.1 \%)$ & $48(41.0 \%)$ & $68(58.1 \%)$ & 0.71 \\
\hline & No Alcohol & $16(13.7 \%)$ & $33(28.2 \%)$ & $49(41.9 \%)$ & \\
\hline 12 & GCS & $10.78 \pm 3.47$ & $14.11 \pm 1.46$ & $13.09 \pm 2.74$ & $<0.01$ \\
\hline 13 & Volume & $39.49 \pm 30.73$ & $15.69 \pm 9.65$ & $23.01 \pm 21.70$ & $<0.01$ \\
\hline 14 & ICH Score & $1.86 \pm 1.13$ & $0.77 \pm 0.84$ & $1.10 \pm 1.06$ & $<0.01$ \\
\hline \multirow[t]{2}{*}{15} & Operated & $14(12.0 \%)$ & $7(6.0 \%)$ & $21(17.9 \%)$ & $<0.01$ \\
\hline & Non Operated & $22(18.8 \%)$ & $74(63.2 \%)$ & $96(82.1 \%)$ & \\
\hline
\end{tabular}


Mean GCS of the population under study was $13.09 \pm 2.74$ and the GCS with pneumonia was 10.78 \pm 3.47 . Lower GCS was found to be strongly associated with occurrence of pneumonia in spontaneous intracerebral hemorrhage $(\mathrm{P}<0.01)$. The mean volume of the intracerebral bleed was $23.01 \pm 21.70 \mathrm{ml}$ and volume in patients with $\mathrm{ICH}$ and pneumonia was $39.49 \pm 30.73 \mathrm{ml}$ $(\mathrm{P}<0.01)$ The mean ICH score was $1.10 \pm 1.06$ and the higher ICH score was significantly associated with the occurrence of pneumonia ( $\mathrm{P}=0.01)$. Twenty-one (17.9 $\%$ patients in our study underwent some form of operative surgeries for the hemorrhage; and $66.66 \%$ (14/21) of operated cases developed pneumonia which was statistically significant $(\mathrm{P}=0.01)$ (Table 1$)$. The location of ICH in our study group was lobar in 41(35\%) cases, ganglionic in $68(58.1 \%)$ cases and brain stem in 7 (6\%) cases. Twenty-three cases (19.7\%) had ventricular extension. Patients with deep seated hematomas like ganglionic, brainstem and those with ventricular extension had significant difference in occurrence of pneumonia in our series (Table 2.) Mean MRS score in our series at discharge was $2.38 \pm 1.99$ and higher score was statistically significantly with occurrence of pneumonia ( $\mathrm{p}=0.01$.). Mortality was seen in $14(12 \%)$ patients in our series, of which $42.86 \%$ (6/14) had pneumonia and 57.14\% (8/14) had no pneumonia $(\mathrm{p}=0.36)$.

The association of our primary exposure variables, such as Diabetes mellitus, COPD, mean GCS, ICH volume, operated status, Ganglionic, Brain stem and Ventricular location of hematoma was statistically significant with the outcome variable i.e. pneumonia. No significant differences were observed regarding demographic data, seizure status, dysarthria, Hypertension status, History of cardiac disease, previous stroke, history of alcohol consumption and ICH associated mortality. Outcome of patients in terms of MRS at discharge was also poorer for patients with pneumonia in Intracerebral hemorrhages $(p=0.01)$ however there was no significant difference in death in patients with pneumonia or no pneumonia in ICH in our study (Table 3).

Table 2. Location of ICH versus Pneumonia

\begin{tabular}{lllll}
\hline $\begin{array}{l}\text { ICH } \\
\text { location }\end{array}$ & $\begin{array}{l}\text { Pneumonia } \\
(\%)\end{array}$ & $\begin{array}{l}\text { No Pneumonia } \\
(\%)\end{array}$ & $\begin{array}{l}\text { Total } \\
(\%)\end{array}$ & P \\
Lobar & & & & 0.16 \\
Yes & $16(13.7$ & $25(21.4)$ & $41(35)$ & \\
No & $20(17.1)$ & $56(47.9)$ & $76(65)$ & \\
Ganglionic & & & & $<0.01$ \\
Yes & $14(12)$ & $54(46.2)$ & $68(58.1)$ & \\
No & $22(18.8)$ & $27(23.1)$ & $49(41.9)$ & \\
Brainstem & & & & 0.03 \\
Yes & $5(4.3)$ & $2(1.7)$ & $7(6)$ & \\
No & $31(26.5)$ & $79(67.5)$ & $110(94)$ & \\
Ventricular & & & & 0.01 \\
Yes & $12(10.3)$ & $11(9.4)$ & $23(19.7)$ & \\
No & $24(20.5)$ & $70(59.8)$ & $94(80.3)$ & \\
\hline
\end{tabular}

\section{Discussion:}

A variety of medical and neurological complications occurs after hemorrhagic stroke, such as pneumonia, urinary tract infections, pressure sores, psychological issues, malnutrition or volume depletion. Many of these complications are potentially preventable with the improvement of stroke care. Pneumonia is among the leading cause of death in the post-acute phase of hemorrhagic stroke. ${ }^{3}$ Pneumonia being a common occurrence after stroke, it significantly contributes to the morbidity and mortality experienced by stroke survivors. Around 6-22\% of patients with stroke will experience pneumonia during the course of treatment. The primary risk factor for pneumonia after hemorrhagic stroke is documented to be dysphagia that allows aspiration of ingested food, liquids, or oral secretions leading to 
aspiration pneumonitis and/or aspiration pneumonia. $^{3}$ Besides aspiration pneumonia, other causes include hypostatic pneumonia secondary to poor caring and immobilization. Routine chest physiotherapy, incentive spirometry, frequent change of position of the patients and mobilization may prevent this type of infection. Thus, a better understanding of the risk factors and comorbid conditions may guide the implementation of strategies in organized hemorrhagic stroke care provision.

According to the study by Jian Liu et al. diabetes mellitus is a strong risk predictor of pneumonia mortality, and especially accounts for an increased incidence of pneumonia in hemorrhagic stroke patients. ${ }^{4}$ Another study from Yang $\mathrm{Li}$ et al. also documents fasting hyperglycemia as an independent risk factor for predicting stroke associated pneumonia. ${ }^{5}$ In our study $13.7 \%$ of patients were admitted to the hospital with concomitant diagnosis of Diabetes Mellitus; $62.5 \%$ of whom developed pneumonia during the hospital stay, and the association was statistically significant $(\mathrm{P}<0.01)$. The presence of COPD is independently associated with higher odds of dying during stroke. The reason for increased mortality in a COPD patients as per the study from Lekoubeu et al. was due to increased incidence of pneumonia in COPD patients than in non- COPD patients with ischemic stroke and intracerebral bleed. ${ }^{6}$ In our observational study, previous history of COPD was found in $20.5 \%$ population and among them $58.33 \%$ patients developed pneumonia during the hospital stay, and the outcome was statistically significant $(\mathrm{P}<0.01)$. VB Id et al. also demonstrated that tobacco smoke exposure is significantly associated with the development of community acquired pneumonia in current smokers and ex-smokers. ${ }^{7}$ In our study, $24.85 \%$ of study population smoked, among that $58.62 \%$ developed pneumonia in their hospital stay; and the result was also statistically significant $(\mathrm{P}<0.01)$.

Table 3. Outcome of ICH according to pneumonia

\begin{tabular}{lllll}
\hline Outcome & $\begin{array}{l}\text { Pneumonia } \\
(\%)\end{array}$ & $\begin{array}{l}\text { No } \\
\text { Pneumonia } \\
(\%)\end{array}$ & Total (\%) & P \\
MRS & $3.14 \pm 2.17$ & $2.04 \pm 1.81$ & $2.38 \pm 1.99$ & 0.01 \\
Death & $6(5.1)$ & $8(6.8)$ & $14(12)$ & 0.36 \\
No Death & $30(25.6)$ & $73(62.8)$ & $103(88)$ &
\end{tabular}

According to study by Ritarwan et al. pneumonia was related with the severity and type of stroke and length of in-hospital stay. The severity of the neurological deficit evaluated by the National Institutes of Health Stroke Scale (NIHSS) was shown to be the only independent risk factor for pneumonia in acute stroke patients. ${ }^{8}$ There was no complete data on NIHSS in our study as it was a retrospective study but our study supports the finding that pneumonia was significantly associated in the population with low GCS, high ICH score, increased intraparenchymal blood volume and those who were operated.

The underlying molecular mechanisms for increased risk of developing pneumonia after ICH need to be further investigated. ${ }^{9}$ In our study there was significant higher MRS score at discharge in patient who developed pneumonia in hospital course showing poorer outcome in such patients in comparison to those not having pneumonia however there was no significant difference in mortality in both the group.

We also found that excess alcohol consumption, deep seated hematomas, hematoma volume, and intraventricular extension were associated with increased risk of pneumonia after ICH. Considerable 
prior studies have detailed the deleterious effect of alcohol abuse on host immune system, as would result in a higher incidence and severity of infection. ${ }^{9}$

Our study has the limitation of being a single-center hospital-based retrospective observational study with small sample size, and the population under study are not representative of the entire population of Nepal. We didn't include data on some determinants that might have influence on the development of pneumonia as the study was retrospective and the variables included were limited to the data available. A well-structured prospective study analyzing various risk factors preferably multicentric in future can open many windows in this common and important aspect of stroke patients.

Conclusions: Diabetes mellitus, COPD, mean GCS, ICH volume, operated status, higher MRS score are associated with increased risk of development of pneumonia in patients with spontaneous intracerebral hemorrhages. Similarly, deep-seated hematomas like ganglionic hematoma, brainstem hematoma and those having intraventricular hemorrhages are also associated with increased risk of pneumonia. Outcome in patients in terms of MRS were also poorer in those who had pneumonia during the course of treatment although there was no difference in overall mortality.

\section{Reference:}

1. Almeida SR, Bahia MM, Lima FO, Paschoal IA, Cardoso TA, Li LM. Predictors of pneumonia in acute stroke in patients in an emergency unit. Arq Neuropsiquiatr. 2015;73(5):415-9. https://doi.org/10.1590/0004-282X20150046.

2. Garner JS, Jarvis WR, Emori TG, Horan TC, Hughes JM. CDC definitions for nosocomial infections, 1988. Am J Infect Control. 1988;16(3):128-40. https://doi.org/10.1016/0196-6553(88)90053-3.
3. Wilson RD. Mortality and cost of pneumonia after stroke for different risk groups. J Stroke Cerebrovasc Dis. 2012;21(1):61-7. https://doi.org/10.1016/j.jstrokecerebrovasdis.2010.05.0 $\underline{02}$.

4. Liu J. Impact of diabetes mellitus on pneumonia mortality in a senior population: results from the NHANES III follow-up study. J Geriatr Cardiol. 2013;10(3):267-71. http://dx.doi.org/10.3969/j.issn.1671-5411.2013.03.005.

5. Li Y, Zhang Y, Ma L, Niu X, Chang J. Risk of strokeassociated pneumonia during hospitalization: predictive ability of combined A2DS2 score and hyperglycemia. BMC Neurol. 2019;19(1):298. https://doi.org/10.1186/s12883-019-1497-x.

6. Lekoubou A, Ovbiagele B. Prevalance and influence of chronic obstructive pulmonary disease on stroke outcomes in hospitalized stroke patients. eNeurologicalSci. 2017:21-24. https://doi.org/10.1016/j.ensci.2016.11.007.

7. Baskaran V, Murray RL, Hunter A, Lim WS, McKeever TM. Effect of tobacco smoking on the risk of developing community acquired pneumonia: A systematic review and meta-analysis. PLoS One. 2019;14(7):e0220204. https://doi.org/10.1371/journal.pone.0220204.

8. K Ritarwan, CA Batubara, R Dhanu. The relationship between pneumonia and Glasgow coma scale assessment on acute stroke patients. 2018 IOP Conference Series: Earth and Environmental Science. 2018;125(1): 012204. https://doi.org/10.1088/17551315/125/1/012204.

9. Ji R, Shen H, Pan Y, Du W, Wang P, Liu G, et al. Risk score to predict hospital-acquired pneumonia after spontaneous intracerebral hemorrhage. Stroke. 2014;45(9):2620-8. https://doi.org/10.1161/STROKEAHA.114.005023. 